\title{
Mensuração da espiritualidade/religiosidade em saúde no Brasil: uma revisão sistemática
}

\author{
Spirituality/religiousity measurement and health in Brazil: \\ a systematic review
}

Samanta Forti (https://orcid.org/0000-0002-8677-4570) ${ }^{1}$

Carlos Augusto Serbena (https://orcid.org/0000-0001-5568-839X) ${ }^{1}$

Alessandro Antonio Scaduto (https://orcid.org/0000-0002-0257-2374) ${ }^{1}$

${ }^{1}$ Departamento de Psicologia, Setor de Ciências Humanas, Universidade Federal do Paraná. Praça Santos Andrade 50, Ala Alfredo Buffren, Centro. 80020-300 Curirtiba

PR Brasil.

forti.samanta@gmail.com

\begin{abstract}
Spirituality/religiousness has been regarded as an important dimension for health studies and services. The present study consists of a systematic review on health and spirituality/religiousness, specifically, instruments for its measurement. In order to do so, we searched for papers using the keywords "scales spirituality religiousness" and "scales spirituality religiousness and health" in CAPES, DOAJ, Lilacs, Pubmed, Scielo and Web of Science, from 2000 to 2017. The search returned 500 studies, from which 35 were selected, considering their originality, availability, language (English or Portuguese), and description of scales for measuring spirituality/religiousness and adapted for the Brazilian culture. From such papers, 16 instruments were identified; the instruments with most studies were the WHOQOL-100 (as well as its reduced version and the SRPB module), the Spiritual Well-Being Scale and the RCOPE scale. We conclude that studies on this field are scarce, for, although many instruments are described, the continuity of their validation is poor.
\end{abstract}

Key words Spirituality, Health, Scales, Systematic review
Resumo A espiritualidade/religiosidade tem sido apontada como uma importante dimensão para a área da saúde. O objetivo desse estudo é apresentar uma revisão sistemática sobre saúde e espiritualidade/religiosidade, como foco em instrumentos para sua mensuração. Buscou-se por trabalhos com as palavras-chave "escalas espiritualidade/ religiosidade" e "escalas espiritualidade/religiosidade e saúde" nas bases de dados CAPES, DOAJ, Lilacs, Pubmed, Scielo e Web of Science, entre 2000 a 2017. Foram encontrados 500 trabalhos dos quais 35 foram selecionados, adotando critérios de originalidade, disponibilidade, lingua (Espanhol, Inglês ou Português) e descrição de escalas específicas de espiritualidade/religiosidade adaptadas para o contexto brasileiro. Dessa amostra foram encontrados 16 instrumentos de mensuração. Os três instrumentos com mais estudos foram o WHOQOL-100 (incluindo sua versão reduzida e o módulo SRPB), a Escala de Bem-estar Espiritual (EBE) e a Escala de Coping Religioso/Espiritual (CRE) e sua versão abreviada (CRE-Breve). Pode-se concluir que ainda há escassos estudos na área, apesar de haver muitos instrumentos de mensuração, há poucos estudos continuados de validação para o contexto brasileiro.

Palavras-chave Espiritualidade, Saúde, Escalas, Revisão de literatura 


\section{Introdução}

Apesar de, no contexto brasileiro atual, se observar diversos debates acerca de temas que envolvem religiosidade e espiritualidade, apenas mais recentemente houve um crescimento no número de estudos sobre a religiosidade/espiritualidade e suas implicações na saúde física e mental dos indivíduos ${ }^{1,2}$.

Embora relacionados entre si, tais conceitos são distintos, e ainda sem consenso no campo da Psicologia. Apesar disso, os estudos sobre esse tema estão relacionados à história da Psicologia da Religião brasileira ${ }^{3}$. No presente trabalho, discute-se a produção científica relativa à mensuração da religiosidade/espiritualidade e sua relação com a área de saúde, tema que, segundo Esperandio e August ${ }^{4}$, vem ganhando destaque no Brasil nos últimos anos, por meio de estudos empíricos quantitativos.

$\mathrm{O}$ conceito de espiritualidade apresenta ao menos dois pontos de vista ${ }^{5}$. No primeiro deles, a espiritualidade é entendida como uma busca pelo significado da vida e o relacionamento com o sagrado/transcendente. Sob um segundo ponto de vista, a espiritualidade se refere a manifestações humanas que buscam a superação de si, ou de obstáculos, no qual não há necessariamente uma ligação com o sagrado.

Para Lucchetti et al. ${ }^{6}$, a espiritualidade é uma busca pessoal de compreensão das questões existenciais humanas, como o sentido da vida, e da morte, bem como de suas relações com o sagrado/transcendente, sendo que esse processo não está necessariamente relacionado com práticas religiosas. Por fim, Hill e Pargament ${ }^{7}$ afirmam que a espiritualidade pode ser compreendida como o "coração e alma" da religião, podendo ser expressa fora, por exemplo, na fé pessoal e por aqueles que não são formalmente religiosos.

Já a religiosidade pode ser compreendida como a adesão a práticas, que o indivíduo acredita e segue, como por exemplo, participação em templo religioso, leitura de livros religiosos e rezas $^{6}$. Para Panzini et al. ${ }^{8}$, a religiosidade se refere à ligação com um sistema de adoração e uma doutrina partilhada com um grupo.

Zangari e Machado 9 explicam que a religião pode ser compreendida como instituição, organizações com discursos e práticas sobre uma realidade transcendente. A religiosidade seria a forma como cada sujeito experiencia a religião e espiritualidade está relacionado ao sentido que se percebe ou que é atribuído à vida. Nesse ponto de vista a espiritualidade pode ter uma relação com a religiosidade ou isso pode não ocorrer.
Os termos religiosidade e espiritualidade, no presente estudo serão tomados a partir de sua utilização como constructos nos instrumentos que se propõem avaliar tais constructos. Cabe lembrar que os mesmos não são incompatíveis, e a tendência em polarizá-los torna-se pouco proveitoso para a pesquisa científica ${ }^{10}$.

\section{Espiritualidade/religiosidade e saúde}

Em 1999, a Organização Mundial da Saúde (OMS) passou a descrever a qualidade de vida como multidimensional, nas dimensões física, psíquica, social e espiritual ${ }^{11}$. A Associação Mundial de Psiquiatria (WPA) afirma que, no campo da saúde, a religiosidade/espiritualidade possui implicações significativas para prevalência, diagnóstico, tratamento, desfechos clínicos e prevenção de doenças ${ }^{12}$.

De modo geral, os profissionais da área da saúde reconhecem o potencial da religiosidade para saúde e bem-estar, não sendo mais possível estudar religiosidade sem relacioná-la à espiritualidade, compreendida como a dimensão do que dá sentido à vida ${ }^{9}$. Atualmente, as pesquisas estão voltadas para o entendimento de processos específicos como o coping religioso, o apego, a tendência humana de buscar razões por trás de uma experiência, entre outros".

$\mathrm{Na}$ mesma direção, diversos trabalhos têm demonstrado que a espiritualidade/religiosidade permite uma elaboração subjetiva e a atribuição de um sentido à vida, que levam a um aumento da motivação para o enfrentamento e superação de crises $^{13}$.

Alguns autores ${ }^{1,2}$ apontam que indivíduos com maior religiosidade/espiritualidade referem melhor bem-estar geral, menores índices de depressão e ansiedade e, menor prevalência no uso e abuso de substâncias psicoativas e comportamento suicida. Ainda, Lucchetti et al. ${ }^{6}$ referem que há uma relação entre crenças, práticas religiosas e saúde física, no qual indivíduos com maior espiritualidade/religiosidade apresentam menor prevalência de doenças coronarianas, hipertensão, menos níveis de pressão arterial, menor prevalência de doenças infecciosas, menores complicações no período pós-operatório e menor índice de mortalidade.

Diante de tais constatações, há um interesse crescente de pesquisadores e profissionais de saúde em compreender melhor tais fenômenos. Entretanto, o maior desafio que se encontra é a correta aferição da religiosidade/espiritualidade por meio de instrumentos confiáveis e válidos no 
contexto brasileiro ${ }^{4,14}$. As principais críticas a tais instrumentos recaem sob a medição incompleta, ou segmentada, dos aspectos da espiritualidade e religiosidade, além da escassez de estudos sobre suas propriedades psicométricas ${ }^{2,4,14}$.

Esforços anteriores nessa direção ${ }^{15}$ referem a existência de mais de 3.000 estudos que relacionam religiosidade/espiritualidade e saúde, listando 20 instrumentos utilizados na pesquisa em saúde no Brasil e descrevendo os sete mais utilizados. Os mesmos autores reforçam que é necessário expandir os estudos para se compreender a diversidade cultural e geográfica do país.

Visto isso, e o fato de o Brasil apresentar-se como um país religioso e heterogêneo, é importante que se proceda com a validação de instrumentos multidimensionais para uso em pesquisa e intervenção no país ${ }^{4,14,16}$.

Neste artigo, são revisados estudos científicos sobre a avaliação da espiritualidade/religiosidade constantes em bases de dados nacionais e internacionais, referentes ao panorama nacional, com o objetivo de avaliar o estado da arte dos instrumentos de mensuração de espiritualidade/religiosidade e suas relações com a saúde.

\section{Método}

O presente estudo consiste numa revisão sistemática, definida como o estudo de forma organizada, da maior quantidade de resultados de pesquisas sobre um tema, buscando explicar as diferenças encontradas ao longo das mesmas ${ }^{17}$. $\mathrm{Na}$ primeira fase do estudo, realizou-se uma busca nas bases de dados CAPES, DOAJ, Lilacs, Pubmed, Scielo e Web of Science, com os termos "escalas espiritualidade/religiosidade e saúde" e "escalas espiritualidade/religiosidade". O intervalo de tempo estabelecido foi com fim em 2017 e sem data de início; ao final da pesquisa, observou-se que os estudos identificados foram publicados entre 2000 e 2017.

Para a segunda fase, os trabalhos foram selecionados utilizando os seguintes critérios: ser um artigo original, estar disponível em formato completo, ter sido publicado em inglês, português ou espanhol, e descrever escalas específicas de espiritualidade/religiosidade adaptadas para o contexto brasileiro. Foram excluídos artigos de revisão bibliográfica que apenas citassem tais escalas e artigos duplicados.

$\mathrm{Na}$ fase seguinte, realizou-se a leitura e análise dos artigos, a fim de listar os instrumentos utilizados, autoria do artigo, ano de publicação e propriedades psicométricas. Nesta fase, foram identificados três instrumentos mais frequentemente utilizados; os mesmos são descritos a seguir, com ênfase em seu histórico e dados sobre sua construção e objetivo.

A Figura 1 descreve as estratégias utilizadas para a busca nas bases de dados referidas, bem como o número de artigos encontrados e incluídos na presente revisão.

\section{Resultados}

A Tabela 1 mostra os dados sobre os trabalhos encontrados em cada base de dados consultada. No total, foram obtidos 500 trabalhos com as palavras-chave utilizadas. Desses, 382 foram descartados após aplicação dos critérios anteriormente descritos e 83 trabalhos foram descartados por duplicidade. Sendo assim, foram avaliados 35 trabalhos, descrevendo o uso de 16 instrumentos de mensuração de espiritualidade/religiosidade, por sua vez apresentados na Tabela 2.

Os instrumentos encontrados nos artigos e o total de trabalhos sobre os mesmos foram: Brief Multidimensional Measure of Religiousness and Spirituality (BMMRS) (um); Daily Spiritual Experience Scale (DSES) (dois); Escala de Atitude Religiosa/Espiritualidade (um); Escala de Avaliação do Bem-estar Espiritual (SWBS)/Escala de Bem-estar Espiritual (EBE) (cinco); Escala de Coping Religioso/Espiritual Abreviada (CRE -Breve)/Escala de Coping Religioso/Espiritual (CRE) (quatro); Escala de Orientações Religiosas (um); Functional Assessment of Chronic Illness Therapy-Spiritual Well-Being FACIT-SP (um); Índice de Religiosidade de Duke (DUREL)/PDUREL (quatro); Inventário de Religiosidade Intrínseca (um); Perspectiva Espiritual de Reed (um); Private and Social Religious Practice Scale (dois); Escala de Religiosidade de Francis (um); Self-rating Scale for Spirituality (SSRS) (três); Treatment Spirituality/Religiosity Scale (TSRS-Br) (um) e WHOQOL-100/WHOQOL-bref e módulo Religiousness and Personal Beliefs (SRPB) (doze). Desses, três instrumentos não foram validados para o contexto brasileiro (Religiosidade de Francis, Perspectiva Espiritual de Reed e Private and Social Religious Practice Scale). Devido a isso, tais instrumentos não serão considerados no presente estudo.

A partir dos dados acima, foi construída a Tabela 2, na qual são listadas informações sobre as escalas encontradas e seu nível de homogeneidade interna, mais especificamente o alfa de 


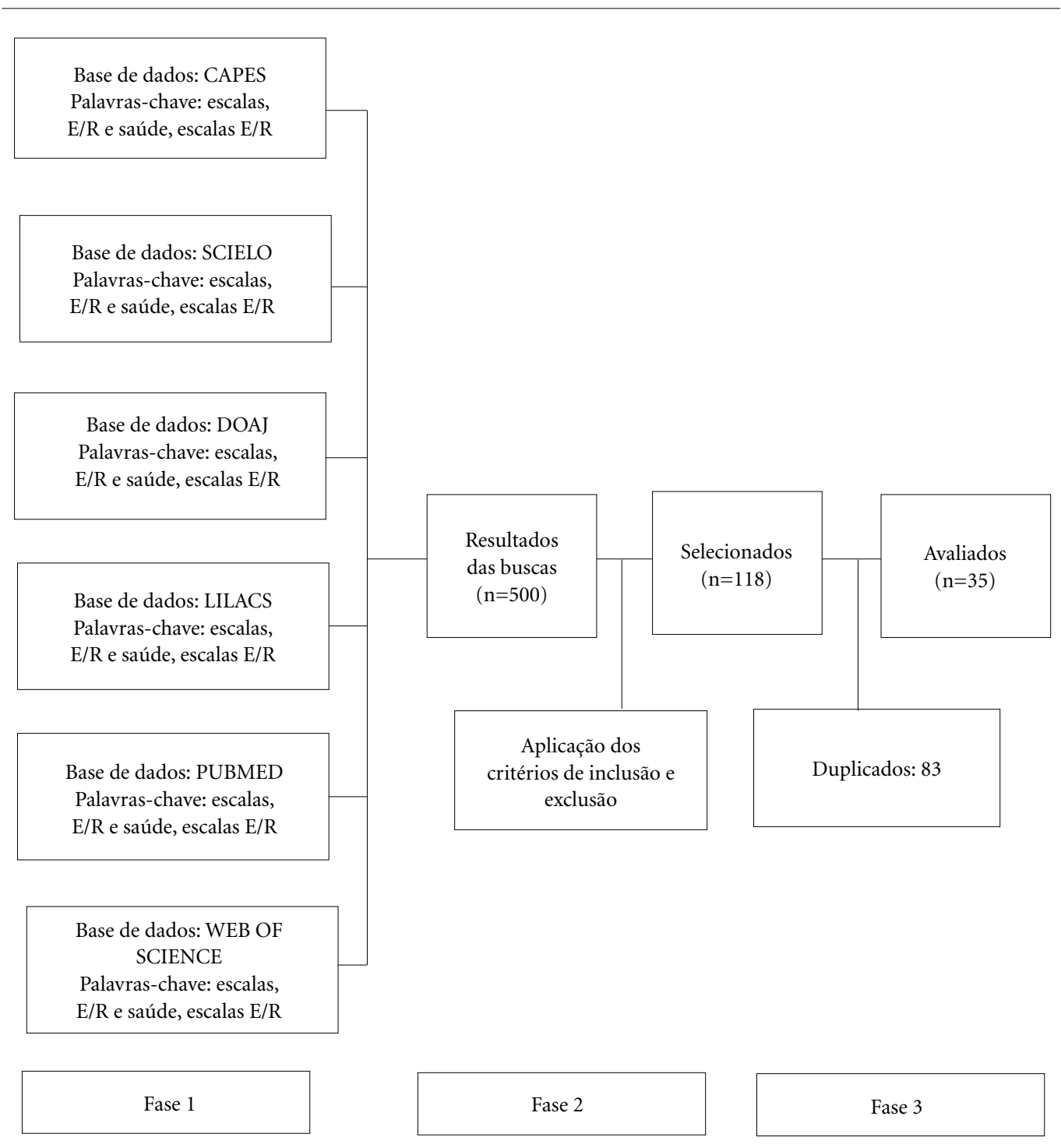

Figura 1. Fluxograma de busca e seleção de artigos nas bases de dados CAPES, SCIELO, DOAJ, PUBMED, WEB OF SCIENCE e LILACS.

Cronbach. Este consiste no índice mais utilizado para avaliação da homogeneidade dos itens de uma escala, permitindo aferir sua fidedignidade ${ }^{18}$. São considerados os seguintes critérios de qualidade dos itens, de acordo com os valores desse coeficiente: (1) excelente $(>0,90),(2)$ bom $(>0,80)$; (3) aceitável $(>0,70)$ e $(4)$ questionável $(>0,60)^{18}$.

Foram avaliados 31 artigos e quatro dissertações de mestrado. As revistas com maior número de publicações foram a Journal of Coloproctology (Rio de Janeiro), Psicologia: Ciência e Profissão, Revista de Psiquiatria Clínica e Ciência e Saúde Coletiva, com duas publicações em cada uma. As publicações dos trabalhos avaliados vão de 2002 a 2017, sendo que o maior número de publicações ocorreu em 2011, 2013, (quatro em cada ano), 2015 (cinco em cada ano) e 2016 (quatro trabalhos em cada ano). Os trabalhos selecionados, foram em sua maioria, publicados em língua portuguesa (19 trabalhos), havendo 12 publicações em inglês e duas em espanhol.

Dos trabalhos analisados, sete são de validações de instrumentos que medem a espiritualidade/religiosidade. Os demais apresentam temas relacionados à espiritualidade e religiosidade com outros aspectos como a qualidade de vida, 
Tabela 1. Trabalhos encontrados com as palavras-chave usadas no presente estudo, por base de dados, entre 2000 a 2017.

\begin{tabular}{|c|c|c|c|c|c|c|c|c|c|c|c|c|}
\hline \multirow[t]{2}{*}{$\begin{array}{c}\text { Palavras- } \\
\text { chave }\end{array}$} & \multicolumn{2}{|c|}{ CAPES } & \multicolumn{2}{|c|}{ DOAJ } & \multicolumn{2}{|c|}{ LILACS } & \multicolumn{2}{|c|}{ PUBMED } & \multicolumn{2}{|c|}{ SCIELO } & \multicolumn{2}{|c|}{$\begin{array}{l}\text { WEB OF } \\
\text { SCIENCE }\end{array}$} \\
\hline & Total & Avaliado & Total & Avaliado & Total & Avaliado & Total & Avaliado & Total & Avaliado & Total & Avaliado \\
\hline $\begin{array}{l}\text { Escalas } \\
\text { de E/R e } \\
\text { saúde }\end{array}$ & 06 & 00 & 08 & 00 & 10 & 10 & 22 & 02 & 01 & 00 & 162 & 07 \\
\hline $\begin{array}{l}\text { Escalas } \\
\text { de E/R }\end{array}$ & 09 & 08 & 05 & 00 & 14 & 04 & 32 & 00 & 02 & 01 & 228 & 03 \\
\hline Total & 15 & 08 & 13 & 00 & 24 & 14 & 54 & 02 & 03 & 01 & 390 & 10 \\
\hline
\end{tabular}

Tabela 2. Escalas de espiritualidade/religiosidade para a cultura brasileira, descritas na literatura entre 2000 a 2017. Base de dados consultadas: CAPES, DOAJ, LILACS, PUBMED, SCIELO e WEB OF SCIENCE.

\begin{tabular}{|c|c|c|}
\hline Escalas & Autores/Ano & Alfa de Cronbach \\
\hline $\begin{array}{l}\text { Brief Multidimensional Measure of } \\
\text { Religiousness and Spirituality (BMMRS) }\end{array}$ & Curcio et al., $2015^{15}$ & $>0,70$ \\
\hline Daily Spiritual Experience Scale (DSES) & $\begin{array}{l}\text { Sánchez et al., } 2014^{19} \\
\text { Kimura et al., } 2012^{20 *}\end{array}$ & $0,91^{* *}$ \\
\hline $\begin{array}{l}\text { Escala de Atitude Religiosa/Espiritualidade, } \\
\text { de Aquino }\end{array}$ & $\begin{array}{l}\text { Aquino et al., } 2009^{21} \\
\text { Aquino, } 2005^{22 \star}\end{array}$ & $0,91^{\star *}$ \\
\hline Escala de Bem-estar espiritual (SWBS) & $\begin{array}{l}\text { Miranda et al., } 2015^{23} \\
\text { Marques et al., } 2009^{24 \star}\end{array}$ & $0,92^{\star *}$ \\
\hline $\begin{array}{l}\text { Escala de Coping Religioso-Espiritual } \\
\text { (CRE) }\end{array}$ & $\begin{array}{l}\text { Silva, } 2016^{25} \\
\text { Panzini, } 2004^{26 *}\end{array}$ & $0,97^{\star *}$ \\
\hline Escala de Orientações Religiosas & $\begin{array}{l}\text { Rodríguez-Rad e Ramos-Hidalgo, } 2017^{27} \\
\text { Ferreira, 2005 } 28 *\end{array}$ & $0,81^{\star *}$ \\
\hline $\begin{array}{l}\text { Functional Assessment of Chronic Illness } \\
\text { Therapy-Spiritual Well-Being (FACIT-SP) }\end{array}$ & $\begin{array}{l}\text { Sánchez et al., 2014 } \\
\text { Lucchetti et al., 2015 } 29 *\end{array}$ & $0,89^{* *}$ \\
\hline Índice de Religiosidade de Duke (DUREL) & Taunay et al., $2012^{30}$ & $>0,80$ \\
\hline Inventário de Religiosidade Intrínseca & Taunay et al., 2012 $31 \star$ & $0,96^{* *}$ \\
\hline Self-rating Scale for Spirituality (SSRS) & $\begin{array}{l}\text { Moreira et al., } 2016^{32} \\
\text { Gonçalves et al., } 2009^{33 *}\end{array}$ & $0,83^{* *}$ \\
\hline $\begin{array}{l}\text { Treatment Spirituality/Religiosity Scale } \\
\text { (TSRS-Br) }\end{array}$ & Gonçalves et al., 2016 $34 \star$ & $0,85^{\star *}$ \\
\hline WHOQOL-SRPB & $\begin{array}{l}\text { Magalhães et al., } 2015^{35} \\
\text { Panzini et al., 2011 }\end{array}$ & $0,96^{* *}$ \\
\hline
\end{tabular}

influência da espiritualidade/religião no enfrentamento de doenças, saúde mental, esperança, depressão, sentido de vida, autoestima, crenças pessoais e morte. Dos instrumentos de mensuração que foram analisados nos artigos, nove medem a espiritualidade, seis a religiosidade, seis a religiosidade/espiritualidade e dois o enfrentamento religioso/espiritual.

O instrumento de mensuração mais utilizado foi o World Health Organization Quality of Life
(WHOQOL-100) ${ }^{37}$, em suas versões completa, reduzida (WHOQOL-Bref) e o módulo WHOQOL -SRPB, que apareceram 12 vezes; a Escala de Bem -Estar Espiritual (EBE), usada em seis trabalhos e a Escala de Coping Religioso-Espiritual (CRE), em suas versões completa e reduzida, usadas em dois trabalhos cada.

A seguir, as escalas citadas no parágrafo anterior serão descritas com mais detalhes. Para fins didáticos, o WHOQOL-100 foi agrupado com 
sua versão reduzida (WHOQOL-bref) e com o módulo de Módulo de Espiritualidade, Religiosidade e Crenças Pessoais (WHOQOL-SRPB). O mesmo foi feito para a Escala de Coping Religioso/Espiritual (CRE), agrupada com sua versão reduzida, a CRE-Breve. Devido às escalas restantes terem sido objeto de apenas uma publicação cada, as mesmas serão abordadas em conjunto num último parágrafo.

$\mathrm{O}$ instrumento mais utilizado para medida da espiritualidade/religiosidade na área da saúde é o World Health Organization-Quality of Life (WHOQOL-100), tendo sido encontrado em 12 dos 35 artigos incluídos para análise. Esse instrumento foi utilizado de forma completa em dois trabalhos analisados; em outros estudos, foram utilizadas apenas algumas de suas subescalas.

O WHOQOL-100 foi desenvolvido por um grupo de especialistas de diversas partes do mundo, reunidos pela Organização Mundial da Saúde (OMS), com objetivo de medir a qualidade de vida; até 2000, já havia sido adaptada para mais de 20 idiomas. O instrumento possui 100 perguntas referentes a seis domínios: físico, psicológico, nível de independência, relações sociais, meio ambiente e espiritualidade/religiosidade/ crenças pessoais ${ }^{37}$.

A versão em português do instrumento foi desenvolvida no Departamento de Psiquiatria e Medicina Legal da Universidade Federal do Rio Grande do Sul, em Porto Alegre, seguindo como método a tradução do instrumento e discussão em grupos focais da versão em português, com objetivo de revisar a formulação e compreensão das 100 perguntas, discutir como essas questões afetam a qualidade de vida de cada sujeito e investigar a possibilidade de outras questões não existentes no questionário, que poderiam ser importantes para o contexto brasileiro. Para tanto, foram realizados grupos focais com pacientes ambulatoriais, pacientes internados, profissionais da área da saúde e pessoas da população geral, todos compostos por sete sujeitos ${ }^{38}$. Após a realização dos grupos focais, foram realizadas mudanças no vocabulário e os autores concluíram que a simplicidade do vocábulo e da estrutura da formulação das questões facilitam a leitura mesmo por pessoas com nível educacional baixo, uma condição para a validade desse instrumento para o contexto brasileiro ${ }^{38}$.

Apesar disso, a necessidade de instrumentos mais curtos, mas com os mesmos parâmetros psicométricos fez com que o Grupo da OMS desenvolvesse uma versão abreviada do WHOQOL-100, o WHOQOL-bref, com 26 questões, composto por quatro domínios: físico, psicológico, relações sociais e meio ambiente ${ }^{38}$. Apesar de a versão breve não conter os domínios "nível de independência" e "espiritualidade", há um item no domínio psicológico onde a espiritualidade é alocada. Posteriormente, a fim de preencher essa lacuna, o Grupo da OMS desenvolveu o Módulo de Espiritualidade, Religiosidade e Crenças Pessoais (WHOQOL -SRPB $)^{8}$. Em nota sobre o significado do WHOQOL-SRPB, Fleck e Skevington ${ }^{39}$ explicam que tal instrumento foi desenvolvido para avaliar como a espiritualidade, religião e crenças pessoais estão relacionadas com a qualidade de vida. Defendem que tais constructos foram incluídos devido a declarações de pacientes, profissionais da saúde e pessoas da comunidade sobre a espiritualidade ser um ponto chave de sua qualidade de vida.

O WHOQOL-SRPB é composto por 32 itens distribuídos em oito facetas, sendo elas: (1) conexão a ser ou força espiritual; (2) sentido na vida; (3) admiração; (4) totalidade e integração; (5) força espiritual; (6) paz interior; (7) esperança e otimismo e (8) fé ${ }^{36}$. A análise fatorial exploratória sugere uma solução de quatro fatores, que explicam $63,5 \%$ da variância. As oito facetas se agrupam nos quatro fatores da seguinte forma: (1) Conexão Espiritual, Fé, Força Espiritual; (2) Paz interior, Totalidade e Integração; (3) Sentido na Vida, Esperança e Otimismo e (4) Admiração. Três facetas apresentaram validade discriminativa para escolaridade, colocando como hipótese que, quanto menos anos de estudo, maior a conexão espiritual, força espiritual e fés ${ }^{36}$. Ainda, em estudo usando o WHOQOL-SRPB, a CRE-breve e o Inventário Beck de Depressão (IDB), Panzini et al. ${ }^{36}$ observaram uma correlação negativa entre nível de depressão e qualidade de vida, embora a conexão espiritual tenha permanecido preservada. Apesar disso, em síntese, o WHOQOL-SRPB apresentou qualidades psicométricas satisfatórias, com relação à validade de constructo, discriminativa $\mathrm{e}$ convergente e à fidedignidade, alfa de Cronbach igual a 0,96 . O mesmo estudo conclui que essa escala está mais ajustada para medir a qualidade de vida, e não a espiritualidade/religiosidade, parecendo ser melhor compreendida como uma medida da relação da religiosidade e crenças pessoais (as quais podem estar ligadas à espiritualidade/ religiosidade) com a qualidade de vida ${ }^{36}$.

A Escala de Bem-Estar Espiritual (EBE) (Spiritual Well-Being Scale) foi desenvolvida por Paloutzian e Ellison em 1982 e adaptada e validada para o contexto brasileiro ${ }^{24}$. A EBE é uma das pioneiras no tema da espiritualidade/religiosidade e foi elaborada com o objetivo de complementar os indicadores de qualidade de vida nos Esta- 
dos Unidos. Foi desenvolvida para avaliar o bem -estar espiritual geral, não sendo fundamentada em assuntos teológicos específicos ou padrões de bem-estar específicos, que podem variar conforme a crença ou religião, sendo um instrumento abrangente ${ }^{40}$.

Essa escala possui 20 itens, divididos em duas subescalas, (1) Bem-estar religioso (BER), que diz respeito à satisfação na conexão pessoal com Deus ou com algo que se considera absoluto, e (2) Bem-estar existencial (BEE), que se refere à percepção do sujeito em relação ao propósito de vida independente de uma referência religiosa ${ }^{24}$. O instrumento é de fácil aplicação e entendimento para variadas idades e condições sociais. Marques et $a .^{24}$, descreveram, uma solução de dois fatores, como a mais apropriada, explicando $47,97 \%$ da variância, com o nível de correlação entre os fatores 1 e 2 de 0,54 , em acordo com os criadores da $\mathrm{EBE}^{24}$. O fator 1 (Bem-estar Religioso) agrupa os itens referentes à relação pessoal com Deus, e se essa relação ajuda a proporcionar bem-estar, satisfação e ausência de solidão, sendo essa fator responsável por $38,41 \%$ da variância total. O fator 2 (Bem-estar Existencial), explicou 9,56\% da variância total, e agrupa itens relacionados à expectativa positiva com relação ao futuro, otimismo e sentimento de propósito na vida. Foram obtidos coeficientes alfa de Cronbach de 0,92 para o fator 1 e 0,85 para o fator 2 , o que sugere uma alta consistência interna ${ }^{24}$.

Apesar disso, a validação da EBE se baseou em amostra apenas da cidade de Porto Alegre, sendo necessários dados de outras regiões, para obter parâmetros comparativos e se chegar a uma padronização para a população brasileira ${ }^{24,41}$, incluindo considerar outras variáveis sociodemográficas para uma melhor contextualização da manifestação da espiritualidade e religiosidade em diferentes grupos. Por fim, Marques et al. ${ }^{24}$ afirmam que, para estudos que visam mensurar a espiritualidade sem as questões religiosas, a EBE não parece adequada. Outro estudo ${ }^{41}$ conclui que há necessidade de novos estudos no contexto brasileiro sobre a EBE, pois entendem que a estrutura fatorial da escala é complexa, assim como encontrado em pesquisas internacionais realizadas por Ledberter et al. ${ }^{42}$, Scott et al. ${ }^{43}$, Miller et al. ${ }^{44}$ e Utsey et al. ${ }^{45}$. O mesmo estudo sugere que o fator Bem Estar religioso é sujeito a um efeito teto em grupos religiosos específicos, uma vez que o indivíduo pode ser influenciado a responder em concordância com os postulados de sua organização religiosa, prejudicando estudos sobre associações entre espiritualidade e saúde ${ }^{41}$.
O terceiro instrumento de mensuração mais citado nos trabalhos encontrados, foi a Escala de Coping Religioso-Espiritual (CRE), adaptada e validada para o contexto brasileiro por Panzini em 2004, a partir da versão original norte-americana (denominada RCOPE Scale) ${ }^{46}$. A CRE é o primeiro instrumento do tipo para o Brasil e foi desenhada para ser teórica e empiricamente embasada, funcionalmente orientada, clinicamente válida, abrangente e considerando aspectos positivos e negativos da religiosidade. Apesar de ter sido estruturada de acordo com a cultura religiosa norte-americana, seus desenvolvedores consideram que sua aplicabilidade é válida em culturas com diferentes crenças e níveis de envolvimento religioso ${ }^{47}$.

A versão final da CRE foi adaptada para o contexto brasileiro ${ }^{26}$ e conta com 87 itens em duas dimensões, o CRE positivo (contendo oito fatores e 66 itens) e o CRE negativo (contendo quatro fatores e 21 itens). Os oito fatores do CRE positivo indicaram uma correlação entre eles que varia de $-0,109$ a 0,420 e foram denominados como: (1) Transformação de si e/ou de Sua Vida, (2) Ações em Busca de Ajuda Espiritual, (3) Oferta de Ajuda ao Outro, (4) Posicionamento Positivo Frente a Deus, (5) Busca Pessoal de Crescimento Espiritual, (6) Ações em Busca do Outro Institucional, (7) Busca Pessoal de Conhecimento Espiritual e (8) Afastamento Através de Deus, da Religião e/ou Espiritualidade. No CRE negativo as correlações dos fatores variaram de $-0,279$ a 0,280 e foram nomeados como: (1) Reavaliação Negativa de Deus, (2) Posicionamento Negativo Frente a Deus, (3) Reavaliação Negativa do Significado e (4) Insatisfação com o Outro Institucional. O alfa de Cronbach da escala CRE $(0,97)$, da dimensão CRE positivo $(0,98)$ e da dimensão CRE negativo $(0,86)$, indicam um ótimo nível de consistência interna, permitindo aplicação clínica e de pesquisa em saúde ${ }^{26}$.

Embora a RCOPE seja uma ferramenta abrangente, ela possui 105 itens (enquanto a CRE ficou com 87), o que limita sua utilização, levando ao desenvolvimento da Brief RCOPE, pelos mesmos autores, com apenas 14 itens ${ }^{47}$. Esta escala, por sua vez, foi traduzida e adaptada para a língua portuguesa ${ }^{48}$ e denominada Escala Breve de Enfrentamento Religioso (CRE-Breve). A versão brasileira, tal como a norte-americana, é composta por dois fatores com sete itens cada (enfrentamento religioso positivo e negativo). Para o fator de enfrentamento religioso positivo, o alfa de Cronbach foi de 0,87 e 27,64\% de variância são explicados pelo mesmo. Esse fator 
contém itens relacionados à religiosidade como fonte de amor, cuidado, força, ajuda, purificação e ressignificação positiva do estressor. O segundo fator (enfrentamento religioso negativo) contém os itens relacionados à religiosidade como fonte de conflito intra e/ou interpessoal, sentimentos de culpa e punição, insegurança e ressignificação negativa do estressor. Esse fator apresentou alfa de Cronbach igual a 0,71 e explicou $15,45 \%$ da variância dos dados. Faria e Seidl ${ }^{48}$ concluem que o instrumento possui estabilidade e adequação para mensuração do constructo enfrentamento religioso.

As escalas encontradas em menor número nos artigos analisados (citadas na Tabela 2) são brevemente descritas a seguir:

- Brief Multidimensional Measure of Religiousness and Spirituality (BMMRS)/Medida Multidimensional Breve de Religiosidade/Espiritualidade (BMMRS-P): é um instrumento multidimensional para medida da espiritualidade e religiosidade para a área da saúde. Possui 38 itens em 11 dimensões (experiências espirituais diárias, valores/ crenças, perdão, práticas religiosas particulares, superação religiosa, apoio religioso, histórico religioso espiritual, comprometimento, religiosidade organizacional, preferências religiosas e autoavaliação global de religiosidade/espiritualidade). $\mathrm{O}$ alfa de Cronbach foi $>0,70$; pela análise fatorial, 11 fatores explicaram $62,84 \%$ da variância total. Os resultados indicam um instrumento breve e multidimensional, confiável e válido para a população brasileira ${ }^{15}$;

- Daily Spiritual Experience Scale (DSES): é um instrumento que busca acessar a dimensão espiritual por meio das experiências que se manifestam na vida diária, como gratidão, compaixão, paz interior, conexão com o transcendente, sem envolvimento com crenças ou comportamentos de uma religião específica. A escala, com 16 itens, apresentou alfa de Cronbach igual a 0,91; a análise fatorial exploratória extraiu três componentes, explicando $60,5 \%$ da variância total, demonstrando índices de fidedignidade e validade aceitáveis ${ }^{20}$;

- Escala de Atitude Religiosa/Espiritualidade: desenvolvida no Brasil ${ }^{22}$, possui 15 itens que agrupam atitudes em três níveis (comportamental, afetivo e cognitivo), relacionadas à espiritualidade e religiosidade, com alfa de Cronbach igual a 0,91;

- Escala de Orientações Religiosas: o objetivo desse instrumento é medir o tipo de orientação religiosa (intrínseca ou extrínseca). A mesma possui dez itens relativos à orientação intrínseca e seis correspondem à orientação extrínseca. $\mathrm{O}$ alfa de Cronbach foi de 0,81 (total), 0,90 para orienta- ção religiosa intrínseca e 0,50 para a orientação extrínseca. A correlação item a item variou entre 0,34 e 0,78 , com média de $0,59^{28}$;

- FACIT-SP: possui 12 itens e avalia o bem -estar espiritual, centrando-se nos aspectos existenciais da espiritualidade e fé e de amplo uso na literatura científica mundial ${ }^{29}$. Foi encontrado um coeficiente alfa de Cronbach de 0,89 para adaptação brasileira desta escala ${ }^{29}$;

- Duke Religious Index (DUREL)/Índice de Religiosidade de Duke (P-DUREL): a escala conta com cinco itens que medem três dimensões da religiosidade, sendo elas: (1) Religiosidade Organizacional, (2) Não Organizacional e (3) Intrínseca. $\mathrm{O}$ alfa de Cronbach $(>0,80)$ e a confiabilidade teste-reteste (Coeficiente de Correlação Intraclasse > $0,90)$, demonstram adequada consistência interna e confiabilidade do instrumento. Apesar de ser uma escala de fácil aplicação, ela aborda apenas três dimensões da religiosidade e não considera a espiritualidade ${ }^{30}$;

- Inventário de Religiosidade Intrínseca: conta com dez itens que almejam a mensurar a religiosidade intrínseca. $\mathrm{O}$ alfa de Cronbach $(0,96)$ e a confiabilidade teste-reteste (Coeficiente de Correlação Intraclasse $>0,70$ ), demonstram adequada consistência interna e confiabilidade do instrumento ${ }^{31}$;

- Self-rating Scale for Spirituality (SRSS): é composta por seis itens relativos à orientação espiritual do indivíduo. O conteúdo dos itens aborda a importância de passar o tempo com pensamentos espirituais e meditações, esforço para viver de acordo com as crenças religiosas, relevância dos pensamentos espirituais que o indivíduo possui, interesse na literatura religiosa, impacto da espiritualidade ao manter o equilíbrio e estabilidade na vida e, consideração que se dá à espiritualidade como base para a vida. $\mathrm{O}$ valor de alfa de Cronbach obtido no estudo brasileiro com o instrumento foi de $0,83^{33}$;

- Treatment Spirituality/Religiosity Scale (TS$R S-B r)$ : contempla 10 itens e mensura a ênfase dada à espiritualidade e religiosidade em tratamentos de usuários de substâncias. $\mathrm{O}$ alfa de Cronbach obtido foi de 0,85 , a confiabilidade teste-reteste apresentou um coeficiente variando entre 0,22 a 0,47 , mostrando-se válida e adequada para o contexto brasileiro ${ }^{34}$.

\section{Discussão}

A análise da produção sobre escalas de espiritualidade/religiosidade demonstrou que ainda há 
poucos trabalhos, ou apenas pesquisas pontuais sobre o tema, ainda que o mesmo venha ganhando forma no campo da pesquisa. A maior parte dos estudos se concentra nas áreas de Psicologia, Enfermagem e Medicina, porém já foram encontradas iniciativas em áreas como Odontologia e Marketing. Apesar disso, o uso dessas escalas é de difícil generalização para população brasileira como um todo até o momento, em virtude de a maior parte dos estudos com as mesmas ter usado amostras apenas da Região Sul do país ${ }^{24,26,36}$.

Ainda, a maior parte dos estudos de validação encontrados se referiu à WHOQOL-SRPB e à CRE e, em menor especificidade, a EBE. Apesar disso, os estudos sobre essa última escala mostraram uma correlação de seus escores com indicadores de bem-estar, incluindo autoconhecimento positivo, sentido e propósito de vida, assertividade e baixa agressividade, o que é de interesse para o uso dessa escala na área de Saúde. O mesmo pode ser dito para a CRE, para o qual foram encontradas correlações significativas com qualidade de vida e saúde, através de suas correlações com os instrumentos Escala de Atitude Religiosa e o WHOQOL-bref. Por fim, os escores da WHOQOL-SRPB apresentaram correlações significativas com qualidade de vida (mas não com saúde mental), o que sugere sua relativa independência como fenômeno de interesse para profissionais de saúde em geral.

A análise das escalas com mais estudos encontrados também apresentou importantes diferenças conceituais. No instrumento WHOQOL-SRPB, não há uma distinção clara entre espiritualidade e religiosidade, o que é justificado pelos autores do instrumento como parte do objetivo do mesmo, qual seja, aferir as interlocuções da espiritualidade/religiosidade com a qualidade de vida, compreendendo que tais fatores são positivamente associados com o nível de saúde e indicam características adaptativas da vida ${ }^{36}$. A Escala EBE traz a diferenciação do conceito de bem-estar espiritual e bem-estar religioso, sendo o primeiro compreendido como o encontro de propósito que justifique o comprometimento com a vida e o segundo, relacionado à comunhão e relação com Deus ou com algo sagrado ${ }^{24}$. Por fim, na CRE, os autores propõem uma relação significativa entre a espiritualidade/religiosidade e estratégias de coping, mostrando que a conexão espiritual e o apoio de membros de uma instituição religiosa colaboram para a mudança de alguns comportamentos, ajudando a enfrentar situações estressantes ${ }^{26}$.

Em comparação com estudo anterior sobre esse tipo de escalas ${ }^{49}$, no qual encontrou-se 20 instrumentos, a pesquisa atual abordou apenas 10 , visto que não foram avaliados instrumentos que não passaram pelo processo de tradução/ adaptação, conforme explicado na metodologia. Ainda, nesse estudo anterior outros instrumentos foram avaliados, os quais não foram encontrados na presente revisão sistemática (escalas de Experiências Espirituais Diárias, Moschella, Prática Religiosa Privada e Social, Autoavaliação de Religiosidade e Motivação Religiosa Intrínseca). Essa diferença sugere diferenças de bases de dados consultadas; em vista de um dos critérios adotados no presente estudo ter sido o de disponibilidade de acesso público dos estudos, acredita-se que a mudança do tipo de acesso a essas escalas pode ampliar seu uso por profissionais de saúde em geral.

\section{Considerações finais}

As evidências sobre a relação entre religiosidade/ espiritualidade e saúde sustentam a importância de investimento na investigação e desenvolvimento de instrumentos efetivos para avaliação dessa dimensão humana. Dos trabalhos analisados, pôde-se observar que os instrumentos disponíveis no contexto brasileiro ainda necessitam de avaliações mais precisas e amostras mais amplas, uma vez que grande parte deles utilizaram uma amostra pouco representativa do país. Além disso, há muitos instrumentos de mensuração, porém com poucos estudos e coesão teórica entre si. 


\section{Colaboradores}

Os autores participaram igualmente da concepção e revisões que resultaram neste artigo.

\section{Referências}

1. Dalgalarrondo P. Relações entre duas dimensões fundamentais da vida: saúde mental e religião. Rev Bras Psiquiatr 2006; 28(3):177-178.

2. Moreira-Almeida A, Lotufo Neto F, Koenig HG. Religiousness and mental health: a review. Rev Bras Psiquiatr 2006; 28(3):242-250.

3. Paiva GJ, Zangari W, Verdade MM, Paula JRM, Faria DGR, Gomes DM, Fontes FCC, Rodrigues CCL, Trovato ML, Gomes AMA. Psicologia da Religião no Brasil: a produção em periódicos e livros. Psic Teor Pesq 2009; 25(3):441-446.

4. Esperandio MRG, August H. A pesquisa quantitativa em Psicologia da religião no Brasil. Revista Pistis Práxis Teologia Pastoral 2017; 9(1):49-67.

5. Pessanha PP, Andrade ER. Religiosidade e Prática Clínica: um olhar fenomenológico-existencial. Perspectivas Online 2009; 3(10):75-86.

6. Lucchetti G, Lucchetti ALG, Avezum, AJ. Religiosidade, Espiritualidade e Doenças Cardiovasculares. Rev Bras Cardiol 2011; 24(1):55-57.

7. Hill PC, Pargament KI. Advances in the conceptualization and measurement of religion and spirituality: Implications for physical and mental health research. Am Psychol 2003; 58(1):64-74.

8. Panzini RG, Rocha N, Bandeira DR, Fleck MPA. Qualidade de vida e espiritualidade. Rev Psiquiatr Clín 2007; 34(1):105-115.

9. Zangari W, Machado FR, organizadores. Psicologia e Religião: Histórico, Subjetividade, Saúde Mental, Manejo, Ética Profissional e Direitos Humanos [cartilha]. São Paulo: Universidade de São Paulo; 2018.

10. Hill PC, Pargament KI, Wood RW, McCullought ME, Swyers JP. Conceptualizing religion and spirituality: points of commonality, points of departure. J Theory Soc Behav 2000; 30(1):51-77.

11. World Health Organization (WHO). Fifty-second World Health Assembly, Geneva, 17-25 May 1999: verbatim records of plenary meetings and list of participants. Genebra: WHO; 1999.

12. Moreira-Almeida A, Sharma A, Van Rensburg BJ, Verhagen PJ, Cook CC. WPA Position Statement on Spirituality and Religion in Psychiatry. World Psychiatry 2016; 15(1):87-88.

13. Koenig HG. Religion and mental health: what should psychiatrists do? BJ Psych Bull 2008; 32(6):201-203.

14. Lucchetti G, Lucchetti ALG, Vallada H. Measuring spirituality and religiosity in clinical research: a systematic review of instruments available in the Portuguese language. Sao Paulo Med J 2013; 131(2):112-122.

15. Curcio C, Lucchetti G, Moreira-Almeida A. Validation of the Portuguese version of the Brief Multidimensional Measure of Religiousness/Spirituality (BMMRS -P) in clinical and non-clinical samples. J Relig Health 2015; 54(2):435-48.

16. Moreira-Almeida A, Pinski I, Zaleski M, Laranjeira R. Envolvimento religioso e fatores sociodemográfico: resultados de um levantamento nacional no Brasil. Rev Psiquiatr Clín 2010; 37(32):3-6.

17. Castro AA, Saconato H, Guidugli F, Clark OAC. Curso de revisão sistemática e metanálise. São Paulo: LEDDIS/UNIFESP; 2002. 
18. Damásio BF. Uso da análise fatorial exploratória em psicologia. Ava Psic 2012; 11(2):213-228.

19. Sánchez R, Sierra F, Zárate K. ¿Son la religiosidad y la espiritualidad dimensiones diferentes? Rev Colomb Cancerol 2014; 18(2):62-68.

20. Kimura M, Oliveira A, Mishima L, Underwood L. Adaptação cultural e validação da Underwood's Daily Spiritual Experience Scale - versão brasileira. Rev Esc Enferm USP 2012; 46(n. esp.):99-106.

21. Aquino T, Correia A, Marques A, Souza, C, Assis H, Araújo IF, Dias PS. Atitude religiosa e sentido da vida: um estudo correlacional. Psicol Cien Prof 2009; 29(2):228-243

22. Aquino T. Atitude religiosa e crenças pessoais dos alunos de psicologia. Revista UNIPÊ, 2005; 9(1):56-63

23. Miranda SL, Lanna MAL, Felippe WC. Espiritualidade, Depressão e Qualidade de Vida no Enfrentamento do Câncer: Estudo Exploratório. Psicol Cien Prof 2015; 35(3):870-885

24. Marques LF, Sarriera JG, Dell'Aglio DD. Adaptação e validação da Escala de Bem-estar Espiritual (EBE): Adaptation and validation of Spiritual Well-Being Scale (SWS). Avaliação Psicológica 2009; 8(2):179-186.

25. Silva VMT. A crença sobre a morte e o coping religioso -espiritual em pacientes internados com doenças crônicas [dissertação]. Uberlândia: Universidade Federal de Uberlândia; 2016.

26. Panzini RG. Escala de Coping Religioso-Espiritual (Escala CRE): tradução, adaptação e validação da Escala RCOPE, abordando relações com saúde e qualidade de vida [dissertação]. Porto alegre: Universidade Federal do Rio Grande do Sul; 2004.

27. Rodríguez-Rad CJ, Ramos-Hidalgo E. Influencia de la religiosidad y la espiritualidad en el comportamiento ético del consumidor. Innovar 2017; 27(65):69-80.

28. Ferreira AM. Religiosidade em alunos e professores portugueses [tese]. Porto (Portugal): Universidade Aberta; 2005.

29. Lucchetti G, Lucchetti ALG, Gonçalves B, Vallada H. Validation of the Portuguese version of the Functional Assessment of Chronic Illness Therapy-Espiritual Well-Being scale (FACIT-Sp 12) among Brazilian psychiatric inpatients. J Relig Health 2015; 54(1):112-121.

30. Taunay TCE, Gondim FAA, Macêdo DS, Moreira-Almeida A, Gurgel LA, Andrade LMS, Carvalho AF. Validação da versão brasileira da escala de religiosidade de Duke (DUREL). Rev Psiquiatr Clín 2012; 39(4):130135.

31. Taunay TC, Cristiano EV, Machado MO, Rola FH, Lima JWO, Macêdo DS, Gondim FAA, Moreira-Almeida, Carvalho AF. Development and validation of the Intrinsic Religiousness Inventory (IRI). Revista Brasileira Psiquiatria 2012; 34(1):76-81.

32. Moreira CNO, Marques CB, Silva MAP, Pinheiro FAM, Salomé GM. Association of sociodemographic and clinical factors with spirituality and hope for cure of ostomized people. J Coloproctol (Rio J) 2016; 36(3):162-172.
33. Gonçalves AM, Pillon SC. Adaptação transcultural e avaliação da consistência interna da versão em português da Spirituality Self Rating Scale (SSRS). Rev Psiquiatr Clin 2009; 36(1):10-15.

34. Gonçalves AMS, Santos MA, Chaves ECL, Pillon SC Adaptação transcultural e validação da versão brasileira da Treatment Spirituality/Religiosity Scale. Rev Bras Enfermagem 2016; 69(2):235-241.

35. Magalhães SM, Carvalho ZMF, Andrade LM, Pinheiro AKB, Studart RMB. Influence of spirituality, religion and beliefs in the quality of life of people with spinal cord injury. Texto Contexto Enferm 2015; 24(3):792800.

36. Panzini RG, Maganha C, Rocha NS, Fleck MPA. Validação brasileira do Instrumento de Qualidade de Vida/espiritualidade, religião e crenças pessoais. Rev Saude Publica 2011; 45(1):153-165.

37. Fleck MPA. O instrumento de avaliação de qualidade de vida da Organização Mundial da Saúde (WHOQOL-100): características e perspectivas. Cien Saude Colet 2000; 5(1):33-38.

38. Fleck MPA, Louzada S, Xavier M, Chachamovich E, Vieira G, Santos L, Pinzon V. Aplicação da versão em português do instrumento abreviado de avaliação da qualidade de vida "WHOQOL-bref". Rev Saude Publica 2000; 34(2):178-183.

39. Fleck MPA, Skevington S. Explicando o significado do WHOQOL-SRPB. Rev Psiquiatr Clín 2007; 34(Supl. 1):146-149.

40. Volcan SMA, Sousa PLR, Mari JJ, Horta BL. Relação entre bem-estar espiritual e transtornos psiquiátricos menores: estudo transversal. Rev Saude Publica 2003; 37(4):440-445

41. Martinez E, Almeida R, Garcia F, Carvalho C. Notas sobre a versão em língua portuguesa da Escala de Bem -Estar Espiritual. J Bras Psiquiatr 2013; 62(1):76-80.

42. Ledbetter MF, Smith LA, Vosler-Hunter WL, Fischer JD. An evaluation of the research and clinical usefulness of the Spiritual Well-Being Scale. J Psychol Theo$\log y$ 1991; 19(1):49-55.

43. Scott EL, Agresti AA, Fitchett G. Factor analysis of the "Spiritual Well-Being Scale" and its clinical utility with psychiatric inpatients. J Sci Stud Relig 1998; 37(2):314-321

44. Miller G, Fleming W, Brown-Anderson F. Spiritual Well-Being Scale ethnic differences between Caucasians and African-Americans. J Psychol Theology 1998; 26(4):358-364.

45. Utsey SO, Lee A, Bolden MA, Lanier Y. A confirmatory test of the factor validity of scores on the spiritual well -being scale in a community sample of African Americans. J Psychol Theology 2005; 33(4):251-257.

46. Pargament K, Koenig H., Perez L. The Many Methods of Religious Coping: Development and Initial Validation of the RCOPE. J Clinical Psychol 2000; 56(4):519543.

47. Pargament K, Feuille M, Burdzy D. The Brief RCOPE: Current psychometric status of a short measure of religious coping. Religions (Basel) 2011;2(1):51-76. 
48. Faria J, Seidl E. Religiosidade, Enfrentamento e Bem -estar Subjetivo em Pessoas Vivendo com HIV/AIDS. Psicol Estud 2006; 11(1):155-164.

49. Curcio C, Lucchetti G, Moreira-Almeida A. Instrumentos de Avaliação de Religiosidade e Espiritualidade. In: Gorenstein C, Wang Y, Hungerbuhler I. Instrumentos de Avaliação em Saúde Mental. Porto Alegre: Artmed; 2016. p. 464-474.

Artigo apresentado em 28/04/2018

Aprovado em 18/08/2018

Versão final apresentada em 20/08/2018 